\title{
DU RÉCIT AU POÈME : USAGES CENDRARSIENS DES PHRASES NOMINALES
}

\author{
Edit BORS \\ Université Catholique Pázmány Péter, Budapest-Piliscsaba
}

\begin{abstract}
En): This paper deals with a specific mix of literary genres in Cendrars's L'or. We focuse on the analysis of lyrical forms in narrative texts, in particular, on the polyfunctionality of sentences which do not include verbs. It is hypothesised that the uses of sentences without verbs in narrative texts represent a minimalist form of poeticity that we try to describe by proposing a certain typology of the roles that these features play in narrative writings.
\end{abstract}

Keywords (En): literary genres; intergenericity; poeticity; sentences without verbs

Mots-clés (Fr) : genres littéraires ; intergénéricité ; poéticité ; phrases sans verbes

\section{Introduction}

Cet article constitue une tentative de définition des paramètres nécessaires à une description stylistique de l'hybridation des genres romanesque et poétique, en prenant comme terrain d'investigation le cas de L'or de Blaise Cendrars. Si L'or nous touche, c'est aussi par son lyrisme qui apparaît, au premier abord, dans une mise en page (espaces blancs) et dans un rythme saccadé évoquant les vers libres. La poétique cendrarsienne représente un topos antirhétorique, comme le dit MEIZOZ (2006), où la qualité littéraire ne relève pas d'une pratique savante, académique, voire inauthentique, mais d'un rapport à l'existence, par l'introduction d'une écriture oralisée, spontanée et vivante. En effet, Cendrars présente, dans L'or, une sensibilité particulière à « l'usage particulier du potentiel linguistique » (GARDESTAMINE, $2001: 56$ ) propre à la langue poétique, notamment à «l'exploitation de virtualités souvent délaissées »(GARDES-TAMINE, 2001:56) qui sont prêtes à accueillir les formes de la poésie moderne.

Nous pensons avec FRÉDÉRIC (1999) que les textes de Cendrars apparaissent souvent comme des cas d'intergénéricité multiple entre poésie et prose. Le caractère poétique repose sur une construction textuelle essentiellement rythmique et sur un certain « type de rapport au réel » (FRÉDÉRIC, 1999 : 29), notamment, sur un emploi massif de procédés de parataxe qui, en effaçant les liens logiques, produisent un effet de liste pour dire le général, l'atemporel et l'universel. Pour rendre compte de l'hétérogénéité générique de L'or, nous aurons recours aux termes, proposés par ADAM et HEIDMANN (2009) et ADAM (2014), d'appartenance et de participation. Un texte relevant généralement de plusieurs genres, il ne s'agit plus de le classer dans une catégorie (son appartenance), mais « d'observer les potentialités génériques qui le traversent - leur participation à un ou plusieurs genres » (ADAM, 2014 : 26). Par conséquent, le texte de Cendrars n'appartiendrait pas uniquement au genre romanesque mais il serait éventuellement en relation étroite avec d'autres genres, comme le poème en prose, le journal intime ou la lettre fictifs. Dans le cas qui nous préoccupe, on voit que l'indication générique est fournie essentiellement par des éléments péritextuels (GENETTE, 1987), tels que le titre, ou la division en chapitres numérotés, alors que la mise en page et le style telles que l'affectivité, l'organisation rythmique, les reprises immédiates, les répétitions aléatoires des 
formes nominales (GARDES-TAMINE et PELIZZA, 2001) semblent introduire une dimension poétique.

C'est dans ce même esprit que Molinié (2001) est amené à distinguer entre le lyrisme d'un texte entier et des passages lyriques à l'intérieur d'une structure hétérogène, et de postuler l'existence de deux faisceaux de traits, l'un étant en rapport étroit avec l'affectivité, l'autre visant un effet de minimalisation : « Il peut donc y avoir un texte lyrique comme tout, ou des passages lyriques, à titre de dominante, dans un texte plus globalement hétérogène. Dans l'un ou l'autre cas, le lyrisme apparaît dans des marques formelles simples : mots de la première personne (pronoms personnels, possessifs, désinences verbales); vocabulaire de l'affectivité ; syntaxe caractérisée par l'importance des mélodies interrogatives ou exclamatives, appuyée éventuellement sur des modalisations d'intensité et des faits d'interruption, ou, au contraire, minimalisation de la structure grammaticale, par insertion de phrases verbales nucléaires au présent, au passé composé ou au futur, ou de phrases nominales, dans une série ouverte ou lâche. Tous ces traits ne sont évidemment significatifs que dans la mesure où ils sont les plus rassemblés : c'est leur densification qui signale le passage lyrique. » (MoLINIÉ, 2001 : 38)

Pour rendre compte du caractère lyrique de $L$ 'or, il conviendra d'étudier ici, en suivant MOLINIÉ (2001), les procédés de minimalisation dus à la polyfonctionnalité des phrases nominales. ADAM (2014 : 30) s'interroge, à juste titre, sur le bien-fondé d'une telle démarche, notamment sur la pertinence de la description d'un genre de discours par des marques linguistiques. La question est donc de savoir si l'apparition de la dimension poétique peut être expliquée par l'accumulation des occurrences de phrases nominales. Sur ce point, nous adopterons les arguments de Adam et Jeanneret (ADAM, 2014 : 30) selon lesquels une marque linguistique est un paramètre à prendre en compte parmi d'autres pour la caractérisation d'un genre donné. Dans notre cas, les usages des phrases nominales, en tant que traits de minimalisation, permettent de qualifier certains aspects de l'hybridation du genre romanesque et poétique.

\section{Réflexions préliminaires sur l'étude des phrases nominales en stylistique}

Depuis la fin du $19^{\mathrm{e}}$ siècle, la langue littéraire française a tendance à se « substantifier » (SMADJA et PIAT, 2009 : 155), autrement dit, à remplacer le verbe par des noms, ou à l'affaiblir au point de le réduire à son rôle grammatical (marques de temps et de la personne), en attribuant le contenu sémantique au nom. Le phénomène du triomphe du nom sur les verbes (SMADJA et PIAT, 2009) est le plus marqué dans le cas des phrases sans verbes qui ont été la première fois analysées, dans le domaine de la littérature, par Marcel Cressot ${ }^{1}$. Cressot relève trois classes de phrases sans verbes. Il distingue, en premier lieu, les phrases de notation ayant pour fonction d'énumérer des objets. Ces phrases de notation, d'après Cressot, ont une valeur cadrative dans la mesure où elles marquent un ancrage temporel ou spatial, pour SMADJA et PIAT (2009) elles renvoient plutôt à une perception visuelle. En revanche, les phrases exclamatives averbales désignent essentiellement l'affectivité ou la subjectivité du locuteur. Viennent enfin les phrases à substantif

\footnotetext{
${ }^{1}$ Cf. La phrase et le vocabulaire de J.-K. Huysmans (1938) cité par SMADJA et Piat (2009: 169).
} 
tiré d'un nom ou d'un adjectif qui semblent aptes « à décrire directement l'événement ou la perception, tels qu'ils se donnent à la conscience » (SMADJA et PIAT, 2009 : 169).

Dans tous les cas, la phrase sans verbe pose un contenu sans apporter d'autres informations pour présenter les éléments, comme le disent SMADJA et PIAT (2009 : 170) « dans toute l'épaisseur de leur être ». Dans cette optique, la phrase de notation est considérée comme un procédé descriptif qui, grâce à « un filtrage de son contenu par une conscience percevante » (SMADJA et PIAT, 2009 : 171), fonde une écriture phénoménologique. La phrase exclamative, par contre, reflète les mouvements d'une psyché, en permettant d'entrer directement dans la conscience du personnage. Cette relation intime de la conscience et du monde s'observe surtout dans la poésie en prose, genre qui, dans L'or de Cendrars, se mêle imperceptiblement à l'écriture romanesque.

On aura vu que la classification présentée ci-dessus repose essentiellement sur des critères syntaxiques élargis de quelques aspects énonciatifs et textuels (valeur cadrative, subjectivité, procédé descriptif). Pour compléter cette première catégorisation, nous proposerons trois types de phrases nominales conformément aux rôles qu'elles remplissent dans la narration : on distinguera ainsi les phrases nominales de description, d'intériorisation et de dramatisation.

\section{Les phrases nominales de description}

Nous nous proposons dans ce qui suit d'étudier les caractéristiques stylistiques des phrases nominales de description. Un premier critère que nous appliquerons sera celui de la valeur cadrative des phrases nominales qui, rappelons-le, contribue à l'ancrage temporel et spatial du récit. Examinons donc (1) sous cet angle.

(1)

«Le port.

Le port de New York.

1834.

C'est là que débarquent tous les naufragés du vieux monde. Les naufragés, les malheureux, les mécontents. Les hommes libres, les insoumis. Ceux qui ont eu des revers de fortune; ceux qui ont tout risqué sur une seule carte ; ceux qu'une passion romantique a bouleversés. Les premiers socialistes allemands, les premiers mystiques russes. Les idéologues que les polices d'Europe traquent ; ceux que la réaction chasse. Les petits artisans, premières victimes de la grosse industrie en formation. Les phalanstériens français, les carbonari, les derniers disciples de Saint-Martin, le philosophe inconnu, et des Écossais. Des esprits généreux, des têtes fêlées. Des brigands de Calabre, des patriotes héllènes. Les paysans d'Irlande et de Scandinavie. Des individus et des peuples victimes des guerres napoléniennes et sacrifiés par les congrès diplomatiques. Les carlistes, les Polonais, les partisans de Hongrie. Les illuminés de toutes les révolutions de 1830 et les derniers libéraux qui quittent leur patrie pour rallier la grande République, ouvriers, soldats, marchands, banquiers de tous les pays, même sud-américains, complices de Bolivar. Depuis la Révolution française, depuis la déclaration de l'Indépendance (vingt-sept ans avant l'élection de Lincoln à la présidence), en pleine croissance, en plein épanouissement, jamais New York n'a vu ses quais aussi continuellement envahis. Les émigrants débarquent jour et nuit, et dans chaque bateau, dans chaque cargaison humaine, il y a au moins un représentant de la forte race des aventuriers.» (CENDRARS, 1997 : 21-22.) 
Cette séquence, qui présente la masse d'immigrants sous forme d'un tableau formé d'éléments juxtaposés apparemment dans une désorganisation totale, correspond à un passage descriptif introduit par un thème-titre nominal. Outre cette première fonction d'ancrage, qui consiste à poser le thème-titre ou le « pivot nominal » (ADAM, 2001: 85) de la description, les phrases nominales constituent ici une série d'énumérations. Il nous semble intéressant de rappeler les travaux de FRÉDÉRIC (1999) qui, en analysant J'ai tué de Cendrars, observe la même technique d'énumération substantivale dont les membres s'organisent en un archilexème (dans notre cas, cet archilexème correspond aux immigrants débarqués à New York) pour esquisser un cadre historique («Les illuminés de toutes les révolutions de 1830 »), social ( Les petits artisans, premières victimes de la grosse industrie en formation ») ou géopolitique ( « Des brigands de Calabre, des patriotes héllènes »). On voit aussi que les informations sont données par le côté nominal, alors que l'élément verbal n'a qu'une fonction secondaire, celle de relier les éléments nominaux qui, par un effet de retardement, relèguent le côté verbal à la fin. Citons en guise d'illustration la phrase relevée dans (1) : « Depuis la Révolution française, depuis la déclaration de l'Indépendance (vingt-sept ans avant l'élection de Lincoln à la présidence), en pleine croissance, en plein épanouissement, jamais New York n'a vu ses quais aussi continuellement envahis. »

Du point de vue textuel, c'est l'agencement des paragraphes qui mérite d'être examiné de plus près. L'exemple (1) est constitué de quatre paragraphes aux proportions inégales : trois paragraphes formés de phrases nominales et un long paragraphe sans structuration typographique. Pourtant cette séquence, bien que non segmentée, présente une structure thématique. Pour rendre compte de son fonctionnement, nous aurons recours aux travaux de GARDES-TAMINE et PELIZZA (1998) qui distinguent des paragraphes thématiques avec changement de thème et avec thème unique. Dans le premier cas, le changement de paragraphe correspond à un changement de thème, et le nouveau thème est lié à celui du paragraphe précédent. Dans le second cas, un même thème est traité dans les paragraphes suivants. Après un parcours rapide du passage, on voit bien que l'exemple (1) appartient à cette deuxième classe. Les trois phrases nominales constituant des paragraphes distincts posent le thème-titre de la description du quatrième paragraphe : le port de New York. Ce dernier paragraphe contient des énumérations qui assurent non seulement son unité structurelle, mais aussi son unité sémantique (FRÉDÉRIC, 1999).

Dans notre deuxième exemple (2), on observe la même technique d'énumération, toutefois, la description de l'environnement y cède la place à la dénomination des personnes aptes à caractériser le lieu :

(2)

«Un jour, il a une illumination. Tous, tous les voyageurs qui ont défilé chez lui, les menteurs, les bavards, les vantards, les hâbleurs, et même les plus taciturnes, tous ont employé un mot immense qui donne toute sa grandeur à leurs récits. Ceux qui en disent trop comme ceux qui n'en disent pas assez, les fanfarons, les peureux, les chasseurs, les outlaws, les trafiquants, les colons, les trappeurs, tous, tous, tous, tous parlent de l'Ouest, ne parlent en somme que de l'Ouest.

L'Ouest.

Mot mystérieux. » (CENDRARS, 1997 : 29) 
L'exemple (2) a ceci de particulier que les énumérations substantivales, qu'on a pu observer dans l'exemple (1), s'apparentent fort ici à une description d'actions (Adam et PetitJean, 1989). Ce type de séquence, bien que constitué d'actions diverses, n'est pas une séquence narrative, car il semble être dépourvu des principes de la progression et de la transformation. Qui plus est, la description d'actions, au lieu d'assurer une évolution chronologique, "insiste sur la convergence et la simultanéité des actions » (FRÉDÉRIC, 1999 : 24).

Se pose maintenant la question de la structure rythmique qui accompagne les énumérations substantivales et la description d'actions : on voit, là encore, des reprises lexicales («tous», « Ouest») et des groupements binaires ${ }^{2}$ (MOUROT, 1960) (« Ceux qui en disent trop comme ceux qui n'en disent pas assez ») qui contribuent à la dimension poétique et musicale de l'œuvre. Parallèlement, le premier paragraphe qui présente une accumulation d'énumérations s'accompagne d'une chute brève (MOUROT, 1960) ${ }^{3}$ formée, elle aussi, de phrases nominales («L'Ouest. Mot mystérieux. »). Ces derniers éléments emphatiques permettent non seulement de promptement terminer la phrase musicale, mais de résumer le paragraphe dans un style concis et dépouillé.

\section{Les phrases nominales d'intériorisation}

Depuis la fin du $19^{\text {e }}$ siècle, la langue littéraire française a non seulement tendance à se « substantifier » (SMADJA et PIAT, 2009 : 155), mais aussi à s'intérioriser (REGGIANI, 2009). L'intériorisation est due, entre autres, à l'emploi du discours indirect libre qui, par une liberté de construction (conservation des modalités d'énonciation - interrogation, exclamation - et des marques de subjectivité) donne une forme expressive à la parole pensive. En revanche, le $20^{\mathrm{e}}$ siècle voit l'épanouissement du discours direct libre qui est une "forme citationnelle, donc immédiatement "parlante" de la "subjectivité littéraire" » (REGGIANI, 2009 : 122). Le discours direct libre est défini par REGGIANI (2009 : 141) « comme une "citation-monstration" à la fois non régie (elle n'est pas introduite par un verbe de parole ou de pensée) et non marquée typographiquement ; à la différence du monologue autonome, il est inséré dans un contexte narratif ». Cette définition restreinte sera nuancée plus tard par REGGIANI (2010) qui souligne l'importance d'un continuum en précisant qu'il existe des degrés de discours direct libre, basés sur les combinaisons possibles des deux variables, notamment, l'attribution explicite de la citation (par un verbe ou lexème spécifique) et son signalement graphique (par des guillemets ou italique). Ainsi obtient-on les quatre configurations du discours direct libre : (a) il peut être explicitement attribué par son contexte immédiat en même temps que graphiquement signalé, (b) il peut être attribué par son contexte immédiat sans être graphiquement signalé, (c) il peut être

\footnotetext{
${ }^{2}$ Une des composantes du rythme est la constitution des groupements binaires, qui, en respectant le principe de la dualité, forment les parties d'une phrase ou les membres d'une période.

${ }^{3}$ La chute brève, qui se trouve en position finale, se fait remarquer par sa brièveté et tout en cassant le rythme naturel de la phrase, produit un effet d'emphase.
} 
graphiquement signalé sans être explicitement attribué par son contexte immédiat, (d) il peut n'être ni attribué par son contexte immédiat ni graphiquement signalé.

Ce que nous proposons d'étudier plus particulièrement, c'est le premier type (a), notamment le signalement graphique accompagné de l'attribution explicite par une phrase averbale, comme dans l'exemple (3) :

(3)

« À l'ombre d'une treille d'Italie et caressant son chien préféré, il songe à faire venir sa famille d'Europe, à indemniser richement ses créanciers, à sa réhabilitation, à l'honneur de son nom et comment doter sa lointaine petite patrie... Douce rêverie.

«Mes trois fils vont venir, ils auront du travail, ce seront des hommes. Et ma fille, comment est-elle ? Tiens, je vais commander un grand piano chez Pleyel à Paris. Il viendra par la piste que j'ai suivie autrefois et s'il le faut à dos d'hommes...Maria...Tous mes compagnons... »

Rêverie.

Sa pipe s'est éteinte. Ses yeux sont perdus au loin. Les premières étoiles s'allument. Son chien ne bouge pas.

Rêverie. Calme. Repos.

C'est la paix. » (CENDRARS, 1997 : 69-70)

Dans cet exemple, les énoncés constituant le discours direct libre sont mis entre guillemets (ce qui correspond au signalement graphique) et l'attribution explicite ne se fait pas par un verbe comme d'habitude, mais par une phrase nominale («Douce rêverie ») précédée de points de suspension. L'attribution peut se faire aussi bien antérieurement que postérieurement, plus rares sont les cas où on observe les deux en même temps. Notre exemple permet d'illustrer cette configuration spéciale où les phrases nominales d'attribution ont pour fonction d'encadrer les paroles pensives citées. Cette configuration est aussi renforcée par la démarcation des paragraphes (GARDES-TAMINE, 1998) liée à l'énonciation : on voit en (3) que la phrase nominale d'attribution peut constituer un paragraphe distinct pour introduire ou pour clore les paroles intérieures citées au discours direct libre.

En revanche, dans l'exemple (4), la phrase nominale n'apparaît pas comme un élément d'attribution, mais elle fait partie intégrante de la parole intérieure citée.

(4)

«Il éclate de rire à la pensée de la niche qu'il va jouer au courrier officiel en arrivant avant lui à Washington et en apportant lui-même la grande nouvelle ! Dieu, quel coup de foudre ! Je vais prendre par les sentiers de la Sierra ; en passant j' annoncerai la chose au père Gabriel. Encore un brave homme. C'est lui qui va être content, et Shanon n'a qu'à bien se tenir. Maintenant ça va barder et nous allons faire la loi chez nous. » (CENDRARS, 1997 : 133)

Ce type de phrase nominale d'intériorisation introduit par une attribution explicite (« il éclate de rire à la pensée »), sans signalement graphique semble exprimer la pensée brute, sous forme de phrase exclamative ( Dieu, quel coup de foudre ! ») ou affirmative («Encore un brave homme »). Dans les deux cas, l'écriture revêt un caractère plutôt minimaliste. Ce style, pour reprendre la définition de ROY (1993 : 12) « est fait d'un vocabulaire simple, de phrases courtes, d'une syntaxe peu compliquée [...]. La narration, qui s'en tient à ce qui est perceptible par les sens, pourrait être qualifiée de phénoménologique. Les actions, la mise en scène, les descriptions sont réduites au minimum. » 


\section{Les phrases nominales de dramatisation}

Dans notre dernier exemple (5), les phrases nominales constituent une unité autonome. À observer la mise en page, on voit que ce texte isolé, placé au milieu de la page et séparé d'espaces blancs en haut et en bas, prépare une rupture, un bouleversement dans le flux narratif.

(5)

«CHAPITRE VII

Rêverie. Calme. Repos.

C'est la paix.

Non. Non. Non. Non. Non. Non. Non. Non.

Non, c'est l'OR !

C'est l'or.

Le rush.

La fièvre de l'or qui s'abat sur le monde.

La grande ruée de 1848, 49, 50, 51 et qui durera quinze ans.

SAN FRANCISCO ! »(CENDRARS, $1997: 71)$

Ce chapitre, contrairement aux précédents, ne ressemble en rien à une écriture romanesque mais évoque l'univers poétique. Les phrases nominales (énumérations, phrases exclamatives, expansions du nom comme GN et relatives) ont pour fonction ici de changer un état de stabilité en un état de déséquilibre. Au point culminant du récit, les phrases nominales produisent un effet de dramatisation qui annonce le déclin et la chute du protagoniste.

\section{Conclusion}

En définitive, en quoi consiste la poéticité des phrases nominales dans la prose cendrarsienne ? Premièrement, c'est un procédé descriptif qui, en juxtaposant des éléments distincts, vise à une présentation exhaustive du paysage ou du milieu décrits. Au lieu de décrire l'environnement, le narrateur nomme les personnes qu'on y trouve. Par conséquent, les informations sont données par le côté nominal, alors que l'élément verbal, s'il n'est pas effacé, n'a qu'une fonction secondaire, celle de relier les éléments nominaux. En outre, ce procédé a pour rôle d'esquisser un cadre historique et un contexte social par l'énumération et la mention des événements de l'histoire sociale du $19^{\mathrm{e}}$ siècle. Deuxièmement, les phrases nominales permettent de reproduire la vie intérieure des personnages, en introduisant des passages écrits au discours direct libre. Grâce à ce procédé, même en l'absence des dialogues, on a accès directement aux pensées du personnage par l'intermédiaire des formes nominales. Troisièmement, les phrases nominales préparent une rupture dans le flux narratif, en transformant les états de stabililité en états de déséquilibre et en apportant de nouveaux éléments à l'intrigue. Au point culminant du récit, qui prépare la chute du personnage, les phrases nominales produisent un effet dramatique. Les phrases nominales, grâce à une forme d'écriture minimaliste, permettent non seulement de condenser le récit de cette histoire véridique, mais par 
la mixité des genres poétiques et romanesques contribuent à une redéfinition des formes littéraires en termes dynamiques et d'interaction.

\section{BIBLIOGRAPHIE}

ADAM Jean-Michel (2001), Les textes. Types et prototypes, Paris, Nathan.

ADAM Jean-Michel (2014), Genres de récits. Narrativité et généricité des textes, Louvain-la-Neuve, Harmattan-Academia.

ADAM Jean-Michel ; HeIDMANN Ute (2009), Le texte littéraire. Pour une approche interdisciplinaire, Louvain-la-Neuve, Academia Bruylant.

ADAM Jean-Michel ; PETITJEAN André (1989), Le texte descriptif, Paris, Nathan.

CENDRARS Blaise (1997), L'or, Paris, Gallimard.

FRÉDÉRIC Madeleine (1999), Palingénésie de la stylistique : intergénéricité et récit de guerre, Le français moderne 1, Paris, Édition CILF, p. 15-34.

GARDES-TAMINE Joëlle (2001), La stylistique, Paris, Armand Colin.

GARDES-TAMine Joëlle ; PElizZA Marie-Antoinette (1998), La construction du texte. De la grammaire au style, Paris, Armand Colin.

GeNETTE Gérard (1987), Seuils. Paris, Seuil.

MEIzOZ Jérôme (2006), Posture et poétique d'un bourlingueur : Cendrars , Poétique 147, Paris, Seuil, p. 297-315.

MoliniÉ Georges (2001), La stylistique, Paris, PUF.

Mourot Jean (1960), Le génie d'un style. Chateaubriand. Rythme et sonorité dans les «Mémoires d'outre-tombe », Paris, Armand Colin.

REGGIANI Christelle (2009), Le texte romanesque : un laboratoire des voix, in : PHILIPPE Gilles ; PIAT Julien (éds.) La langue littéraire. Une histoire de la prose en France de Gustave Flaubert à Claude Simon, Paris, Fayard, p. 120-154.

REGGIANI, Christelle (2010), Les discours directs libres dans la prose narrative de Stendhal, in : Neveu F. [et al.] (éds.) Congrès Mondial de Linguistique Française, Linguistique du texte et de l'écrit, stylistique, Paris, Institut de Linguistique Française, p. 32-55. En ligne :

http://www.linguistiquefrancaise.org/articles/cmlf/abs/2010/01/cmlf2010_000 040/cmlf2010_000040.html.

Roy Alain (1993), L'art du dépouillement (l'écriture minimaliste), Liberté 35, Montréal, Collectif Liberté, p. 10-28. En ligne :

http://id.erudit.org/iderudit/31505ac.

SMADJA Stéphanie ; PIAT Julien (2009), Le triomphe du nom et le recul du verbe, in : PHILIPPE Gilles ; PIAT Julien (éds.), La langue littéraire. Une histoire de la prose en France de Gustave Flaubert à Claude Simon, Paris, Fayard, p. 155177. 